14. E. W. Weisstein, Pi formulas-From MathWorld, A Wolfram Web Resource, http://mathworld. wolfram.com/PiFormulas.html.

\title{
New Proofs of Euclid's and Euler's Theorems
}

\section{Juan Pablo Pinasco}

In this note we give a new proof of the existence of infinitely many prime numbers. There are several different proofs with many variants, and some of them can be found in $[\mathbf{1}, \mathbf{3}, \mathbf{4}, \mathbf{5}, \mathbf{6}]$. This proof is based on a simple counting argument using the inclusionexclusion principle combined with an explicit formula. A different proof based on counting arguments is due to Thue (1897) and can be found in [6] together with several generalizations, and a remarkable variant of it was given by Chaitin [2] using algorithmic information theory. Moreover, we prove that the series of reciprocals of the primes diverges. Our proofs arise from a connection between the inclusion-exclusion principle and the infinite product of Euler.

Let $\left\{p_{i}\right\}_{i}$ be the sequence of prime numbers, and let us define the following recurrence:

$$
a_{0}=0, \quad a_{k+1}=a_{k}+\frac{1-a_{k}}{p_{k+1}} .
$$

Let us note that the $N$ th term $a_{N}$ generated by this recurrence coincides with

$$
a_{N}=\sum_{i} \frac{1}{p_{i}}-\sum_{i<j} \frac{1}{p_{i} p_{j}}+\sum_{i<j<k} \frac{1}{p_{i} p_{j} p_{k}}-\cdots+(-1)^{N+1} \frac{1}{p_{1} \ldots p_{N}},
$$

and can be given in a closed form as

$$
a_{N}=1-\prod_{i=1}^{N}\left(1-\frac{1}{p_{i}}\right)
$$

which implies that $0<a_{N}<1$, since each factor is strictly positive and less than one.

Now, we are ready to prove the classical Euclid's theorem:

Theorem 1. There are infinitely many prime numbers.

Proof. Let us suppose that $p_{1}<p_{2}<\cdots<p_{N}$ are all the primes. For any $x \geq 1$, and for $i=1, \ldots, N$, let $A_{i}$ be the set of integers in $[1, x]$ that are divisible by $p_{i}$. Then, the number of positive integers in $[1, x]$ is obtained by applying the inclusionexclusion formula to find the cardinality of $\cup_{i=1}^{N} A_{i}$ :

$[x]=1+\sum_{i}\left[\frac{x}{p_{i}}\right]-\sum_{i<j}\left[\frac{x}{p_{i} p_{j}}\right]+\sum_{i<j<k}\left[\frac{x}{p_{i} p_{j} p_{k}}\right]-\cdots+(-1)^{N+1}\left[\frac{x}{p_{1} \cdots p_{N}}\right]$, 
where $[s]$ denotes the integral part of $s$ as usual. Since

$$
\lim _{x \rightarrow \infty} x^{-1}\left[\frac{x}{t}\right]=\frac{1}{t}
$$

we reach a contradiction,

$$
1>a_{N}=\sum_{i} \frac{1}{p_{i}}-\sum_{i<j} \frac{1}{p_{i} p_{j}}+\sum_{i<j<k} \frac{1}{p_{i} p_{j} p_{k}}-\cdots+(-1)^{N+1} \frac{1}{p_{1} \ldots p_{N}}=1,
$$

and the proof is finished.

Let us observe from the previous proof that the asymptotic density $D\left(p_{1}, \ldots, p_{N}\right)$ of the set of integers divisible by none of $p_{1}, \ldots, p_{N}$ is exactly

$$
D\left(p_{1}, \ldots, p_{N}\right)=1-\sum_{i} \frac{1}{p_{i}}+\sum_{i<j} \frac{1}{p_{i} p_{j}}-\sum_{i<j<k} \frac{1}{p_{i} p_{j} p_{k}}+\cdots(-1)^{N} \frac{1}{p_{1} \ldots p_{N}},
$$

that is,

$$
1-a_{N}=D\left(p_{1}, \ldots, p_{N}\right)=\prod_{j=1}^{N}\left(1-\frac{1}{p_{j}}\right)
$$

and let us define $D=\lim _{N \rightarrow \infty} D\left(p_{1}, \ldots, p_{N}\right)$. Then, by taking logarithms, we obtain that

$$
\sum_{p} \ln \left(1-\frac{1}{p}\right)
$$

converges if $D>0$ and diverges if $D=0$. Since $\sum_{p} \frac{1}{p}$ converges if and only if $\sum_{p} \ln \left(1-\frac{1}{p}\right)$ does, it is enough to show that $D=0$ to obtain:

Theorem 2. The series $\sum_{p} \frac{1}{p}$ diverges.

Proof. Let us show that $D>0$ and the convergence of $\sum_{p} \frac{1}{p}$ cannot hold simultaneously. To this end, let us take $0<\varepsilon<D$, and choose $N$ big enough so that

$$
\varepsilon<D\left(p_{1}, \ldots, p_{N}\right) \text { and } \sum_{p>p_{N}} \frac{1}{p}<\varepsilon .
$$

Now the asymptotic density of the integers which are not divisible by any of the primes $p_{1}, \ldots, p_{N}$ is bounded below by $\varepsilon$. However, those integers must be divisible by some prime $p>p_{N}$, so their density is bounded above by

$$
\sum_{p>p_{N}} \frac{1}{p}<\varepsilon,
$$

a contradiction. Hence $D=0$ and the series diverges.

ACKNOWLEDGEMENTS. I want to thank I. Drelichman, R. Durán, P. de Napoli, and L. Vendramin for their help. Also, I want to thank the anonymous referees for their suggestions. This work has been supported by ANPCyT PICT No. 03-05009. 
1. M. Aigner and G. Ziegler, Proofs from THE BOOK, Springer-Verlag, Heidelberg, 1998.

2. G. J. Chaitin, Toward a mathematical definition of life, in The Maximum Entropy Formalism, R. D. Levine and M. Tribus, eds., MIT Press, Cambridge, 1979, pp. 477-498.

3. L. E. Dickson, History of the Theory of Numbers, vol. 1, Chelsea Publishing, New York, 1952, pp. 413415.

4. G. H. Hardy and E. M. Wright, An Introduction to the Theory of Numbers, Oxford University Press, London, 1954, pp. 12-17.

5. W. Narkiewicz, The Development of Prime Number Theory: From Euclid to Hardy and Littlewood, Springer Monographs in Mathematics, Springer-Verlag, Berlin, 2000, pp. 1-10.

6. P. Ribenboim, The New Book of Prime Number Records, Springer-Verlag, New York, 1996, pp. 3-11.

Instituto de Ciencias, Universidad Nacional de General Sarmiento,

J. M. Gutierrez 1150-Los Polvorines (1613), Buenos Aires, Argentina

jpinasco@dm.uba.ar

\section{A Note on Covering a Square of Side Length $2+\epsilon$ with Unit Squares}

\section{Janusz Januszewski}

In [1] Soifer posed the following problem: "Find the smallest number $\Pi(n)$ of unit squares that can cover a square of side length $n+\epsilon$ [for some $\epsilon>0$ ]." For small values of $n$ the estimates presented in [1] are: $5 \leq \Pi(2) \leq 7$ and $10 \leq \Pi(3) \leq 14$. The aim of this paper is to improve the lower bounds. We show that $\Pi(2) \geq 6$ and $\Pi(3) \geq 11$, i.e., we show that it is impossible to cover a square of side length greater than 2 with five unit squares and it is impossible to cover a square of side length greater than 3 with ten unit squares.

Lemma. Let $S$ be a square of side length 1 , let $\delta>0$, and let $l_{1}$ and $l_{2}$ be straight lines parallel to each other with distance $1+\delta$. Moreover, let both $l_{1}$ and $l_{2}$ have a nonempty intersection with $S$. Denote by $s_{i}$ the length of $l_{i} \cap S$ for $i \in\{1,2\}$. Then $s_{1}+s_{2}<1$.

Proof. $S$ has a nonempty intersection with $l_{1}$ and $l_{2}$. Therefore no side of $S$ is parallel to $l_{1}$. We can assume that $l_{1}$ is on the left side of $l_{2}$ as in Figure 1 . Denote by $a$ the vertex of $S$ that lies on the right side of $l_{2}$ (obviously, there is only one such vertex). Let $p_{1}$ denote the distance between $l_{2}$ and $a$ and let $w_{1}$ denote the length of the longest segment which is contained in $S$ and is parallel to $l_{2}$ and whose distance to $a$ equals $1+\delta$. It is easy to see that $w_{1}=s_{1}+s_{2}$ (see Figure 1 ). Let $w$ denote the length of the longest segment which is contained in $S$ and is parallel to $l_{2}$ and whose distance from $a$ equals 1 . From $\delta>0$ we deduce that $w_{1}<w$. To find $w$ observe that $s_{3}=\tan \frac{\alpha}{2}$ and $1-s_{3}=w \cos \alpha$ in Figure 1. Consequently,

$$
w=\left(1-\tan \frac{\alpha}{2}\right) \frac{1}{\cos \alpha}=\left(1-\tan \frac{\alpha}{2}\right) \frac{1+\tan ^{2} \frac{\alpha}{2}}{1-\tan ^{2} \frac{\alpha}{2}}=\frac{1+\tan ^{2} \frac{\alpha}{2}}{1+\tan \frac{\alpha}{2}} .
$$

This value is smaller than 1 , because $\tan \frac{\alpha}{2}<1$ for $0^{\circ}<\alpha<90^{\circ}$. Thus $s_{1}+s_{2}=$ $w_{1}<w<1$. 